\title{
Jahn-Teller Distortion in the Phosphorescent Excited State of Three-Coordinate Au(I) Phosphine Complexes
}

\author{
Khaldoon A. Barakat, Thomas R. Cundari, ${ }^{*}$ and Mohammad A. Omary ${ }^{*}$ \\ Department of Chemistry, University of North Texas, Denton, Texas 76203-5070
}

Received June 4, 2003; E-mail: tomc@unt.edu; omary@unt.edu

\begin{abstract}
Luminescent transition metal complexes have been receiving increasing attention due to their role in a variety of optoelectronic applications. For example, molecular light-emitting devices (LEDs) have been increasingly reported ${ }^{1}$ to exhibit superior efficiencies when phosphorescence is enhanced by the presence of a heavy metal because of spin-orbit coupling. ${ }^{2}$ Other complexes have been suggested as selective chemical sensors for volatile organics, ${ }^{3}$ oxygen, ${ }^{4}$ and specific ions ${ }^{5}$ because the phosphorescence is reversibly enhanced or quenched on interaction with these species. ${ }^{6}$ An understanding of the nature of the luminescent excited state, in addition to its fundamental significance, is essential for designing new materials with improved properties for such applications.
\end{abstract}

Monovalent gold complexes represent one of the most celebrated classes of luminescent complexes. Gold(I) complexes most commonly exist as two-coordinate $\left(\mathrm{AuL}_{2}\right)$ species, but three- $\left(\mathrm{AuL}_{3}\right)$, and four-coordinate $\left(\mathrm{AuL}_{4}\right)$ species also exist. ${ }^{7}$ These species differ in the presence of Au-based luminescence. ${ }^{6}$ While Au-based luminescence is usually absent in $\mathrm{AuL}_{4}$ complexes, it exists in $\mathrm{AuL}_{2}$ complexes only in the presence of $\mathrm{Au} \cdots \mathrm{Au}$ interactions. Meanwhile, $\mathrm{AuL}_{3}$ complexes exhibit Au-based luminescence both with and without $\mathrm{Au} \cdots \mathrm{Au}$ interactions present.$^{8-10}$ Reports by $\mathrm{Gray}^{8}$ and Fackler ${ }^{9}$ on the photophysical properties of monomeric $\left[\mathrm{AuL}_{3}\right]^{+}$ complexes illustrated visible luminescence with very large Stokes' shifts (typically $10000 \mathrm{~cm}^{-1}$ ), which suggests a significant excitedstate distortion. These authors suggested that on the basis of extended Hückel calculations and experimental spectral observations, the Stokes' shift is a result of a $\mathrm{M}-\mathrm{L}$ bond shortening due to excitation from an antibonding HOMO to a bonding LUMO. More accurate information is obtained by fully optimizing the geometry of the excited-state independently because the excited molecule (exciton) should be dealt with as a different entity that is distinct from the ground-state molecule. ${ }^{11}$ The present work provides a dramatic illustration of this for $\left[\mathrm{AuL}_{3}\right]^{+}$complexes $(\mathrm{L}=$ phosphine).

The calculations herein were of two types: QM (quantum mechanical) and QM/MM (hybrid quantum mechanical/molecular mechanical). A general description of the calculations is given below ${ }^{12}$ while full details are available in the Supporting Information, including tests done with a variety of basis sets and different levels of theory to calibrate the computational accuracy. For QM calculations $\left[\mathrm{Au}\left(\mathrm{PH}_{3}\right)_{3}\right]^{+}$was used as a model, and different methodologies, including ab initio and DFT, and basis sets with various complexities were employed. The geometries of all models were fully optimized in both the ground and excited electronic states, with the latter being the lowest triplet state because the experimental lifetime data suggested that the emitting state is phosphorescent. ${ }^{8,9}$ For QM/ MM calculations, $\left[\mathrm{AuL}_{3}\right]^{+}$models were used with $\mathrm{L}=\mathrm{PMe}_{3}, \mathrm{PPh}_{3}$, $\mathrm{PPhCy}_{2}$, and TPA (tris(1,3,5-triaza-7-phosphaadamantane)).

The DFT calculations for the singlet ground state $\left({ }^{1} \mathrm{~A}_{1}{ }^{\prime}\right)$ of $[\mathrm{Au}-$ $\left.\left(\mathrm{PH}_{3}\right)_{3}\right]^{+}$reproduced the electronic structure proposed by Gray and Fackler, with the HOMO being the degenerate e' orbital, which has a predominant $\mathrm{Au} 5 \mathrm{~d}_{x y}, 5 \mathrm{~d}_{x^{2}-y^{2}}$ contribution with an antibonding

14228 a J. AM. CHEM. SOC. 2003, 125, 14228-14229

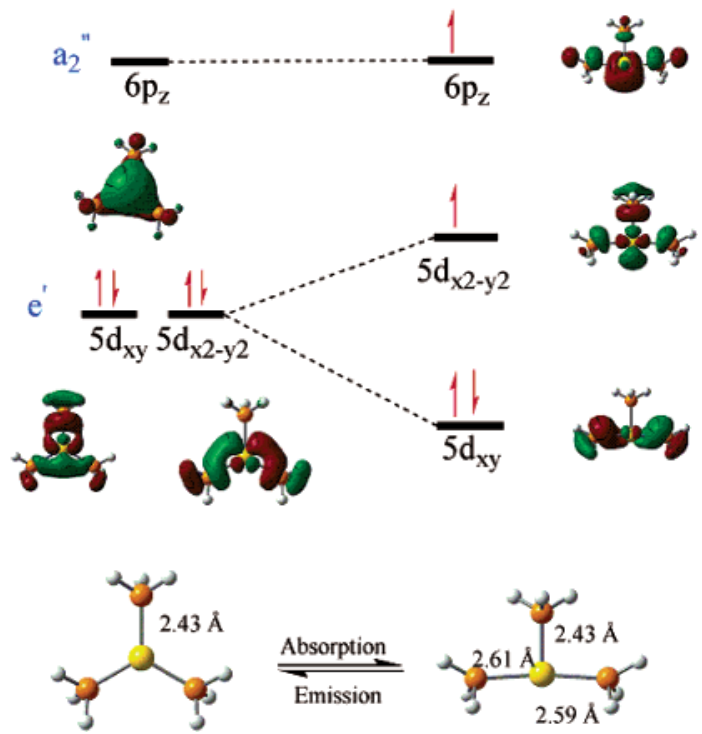

Figure 1. Molecular orbital diagrams (top) and optimized structures (bottom) for the ${ }^{1} \mathrm{~A}_{1}$ ' ground state (left) and the lowest triplet exciton (right) of $\left[\mathrm{Au}\left(\mathrm{PH}_{3}\right)_{3}\right]^{+}$.

AuP character, and the LUMO being the $\mathrm{a}_{2}{ }^{\prime \prime}$ orbital, which has a predominant $\mathrm{Au} 6 \mathrm{p}_{z}$ contribution with a bonding AuP character (Figure 1, left). Calculations for the lowest triplet excited state $\left({ }^{3} \mathrm{E}^{\prime \prime}\right)$ while maintaining the trigonal planar $\left(D_{3 h}\right)$ ground-state geometry performed by a scan of AuP distances for triplet $\left[\mathrm{Au}\left(\mathrm{PH}_{3}\right)_{3}\right]^{+}$gave rise to shorter AuP bonds ( $2.38 \AA$ ) than those in the global minimum for the singlet ground state $(2.43 \AA)$, as expected. However, when the triplet structure is fully optimized without symmetry constraint, the global minimum has a T-shaped structure (Figure 1). The AuP bond distances increased rather significantly for the two trans bonds of the optimized T-shaped triplet excited state compared to the singlet ground state.

A scan of the PAuP angles was carried out, from the trigonal planar $\left(\mathrm{PAuP}=120^{\circ}\right)$ to the $\mathrm{T}$-shaped geometry $\left(\mathrm{PAuP}=90^{\circ}\right.$ and $180^{\circ}$ ). The singlet-triplet transition was calculated at each point along this reaction coordinate. The results showed that when the geometry changes toward the T-shape, the singlet-triplet gap decreases, leading to longer phosphorescence wavelengths $\left(\lambda_{\mathrm{em}}=\right.$ $269 \mathrm{~nm}$ for $\mathrm{PAuP}=120^{\circ}$ and $416 \mathrm{~nm}$ for $\mathrm{PAuP}=90^{\circ}, 180^{\circ}, 90^{\circ}$ ), which supports the large experimental Stokes' shift. In contrast, scan calculations in which the gold geometry was constrained to trigonal planar while varying the AuP distances showed only small changes in the singlet-triplet gap over a wide range of AuP bond lengths. Indeed, compressing the AuP bonds from the ground-state distance of $2.43 \AA$ to $2.38 \AA$ shifts $\lambda_{\text {em }}$ from 268 to $273 \mathrm{~nm}$ while experimental values are in the visible region. 8,9

It is fair to question whether the trigonal to T-shape distortion will take place for larger, more sterically hindered R groups. All 


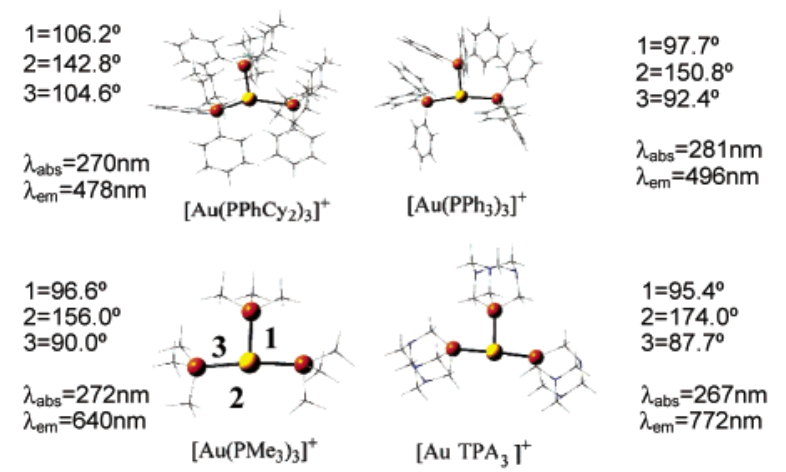

Figure 2. QM/MM-optimized structures of triplet $\left[\mathrm{Au}\left(\mathrm{PR}_{3}\right)_{3}\right]^{+}$models and calculated absorption and emission wavelengths. Note the correlation between $\lambda_{\mathrm{em}}$ and the deviation of angle 2 from linearity.

calculations done in this work, whether $\mathrm{QM}\left(\mathrm{L}=\mathrm{PH}_{3}\right)$ or $\mathrm{QM} /$ $\mathrm{MM}\left(\mathrm{L}=\mathrm{PMe}_{3}, \mathrm{TPA}, \mathrm{PPh}_{3}, \mathrm{PPhCy}_{2}\right)$, showed that the optimized triplet excited state was distorted from trigonal planar toward T-shape. The optimized structures for all models are shown in Figure 2 with additional metric data in the Supporting Information. The geometries of triplet $\left[\mathrm{Au}\left(\mathrm{PR}_{3}\right)_{3}\right]^{+}$deviate further from an ideal T-shape (i.e., PAuP $=90^{\circ}, 180^{\circ}, 90^{\circ}$ ) for bulkier ligands. The deviation from the T-shape is a response to the $\mathrm{R}-\mathrm{-}-\mathrm{R}$ repulsion among the cis-phosphines.

The absorption and emission energies (vertical transitions) were calculated for the fully optimized models. QM calculations ( $\mathrm{L}=$ $\left.\mathrm{PH}_{3}\right)$ gave (absorption, emission) wavelengths of $(269,901 \mathrm{~nm})$. The hybrid QM(B3PW91)/MM calculations with $\mathrm{L}=\mathrm{TPA}, \mathrm{PMe}_{3}$, $\mathrm{PPh}_{3}$, and $\mathrm{PPhCy}_{2}$ gave absorption wavelengths of 267, 272, 281, and $270 \mathrm{~nm},{ }^{13}$ respectively, which are comparable to those obtained with the QM calculations for the model with $\mathrm{L}=\mathrm{PH}_{3}$. The emission wavelengths were 772, 640, 496, and $478 \mathrm{~nm}$, respectively. Thus, proceeding toward bulkier ligands attains higher emission energies. Interestingly, we note that the same trend is followed experimentally from the few reports available ${ }^{8,9}$ for $\left[\mathrm{AuL}_{3}\right]^{+}$species: while the complex with the sterically unencumbered TPA ligand gave an emission maximum of $517-547 \mathrm{~nm}, \lambda_{\mathrm{em}}{ }^{\max }$ values of 512,508 , and $492-513 \mathrm{~nm}$ were obtained for the bulkier complexes with $\mathrm{L}$ $=\mathrm{PPh}_{3}$, dcpe, and TPPTS, respectively. ${ }^{14}$ The results herein clearly indicate that the rearrangement to a T-shape leads to visible emissions and large Stokes' shifts comparable to the experimental data. Calculations that do not incorporate this rearrangement cannot account for the large Stokes' shifts. The computational data suggest that changing the $\mathrm{R}$ substituents affected the emission more than the absorption, indicating a larger effect of the R group on the triplet state of $\left[\mathrm{Au}\left(\mathrm{PR}_{3}\right)_{3}\right]^{+}$. Thus, the presence of a sterically encumbered group leads to a blue-shifted emission with a smaller Stokes' shift as bulky $\mathrm{R}$ groups move the triplet state geometry further away from an idealized $\mathrm{T}$-shape. The correlation between the emission energy and the deviation from the T-shape (Figure 2) is unmistakable.

The geometry change in the excited state of $\left[\mathrm{Au}\left(\mathrm{PR}_{3}\right)_{3}\right]^{+}$species is a Jahn-Teller distortion. Upon photoexcitation, one electron is promoted from the degenerate $\mathrm{e}^{\prime}\left(5 \mathrm{~d}_{x y}, 5 \mathrm{~d}_{\left.x^{2}-y^{2}\right)}\right.$ orbital to the $\mathrm{a}_{2}{ }^{\prime \prime}$ $\left(6 \mathrm{p}_{z}\right)$ orbital, leaving three electrons in $\mathrm{e}^{\prime}$ and rendering the complex Jahn-Teller unstable. One member of the $\mathrm{e}^{\prime}$ pair will be destabilized while its counterpart is stabilized (Figure 1). As the geometry changes from trigonal planar to T-shape, the energy gap between $\mathrm{a}_{2}{ }^{\prime \prime}$ and the higher-energy member of the erstwhile $\mathrm{e}^{\prime}$ decreases. The resulting shift to a longer emission wavelength as the T-shape distortion evolves explains both the emissive triplet state and the direction of the Stokes' shift.
Hoffmann and co-workers ${ }^{15}$ mentioned that a distortion from a 3-fold symmetric geometry to T-shape is feasible for the model trivalent complex $\left(\mathrm{CH}_{3}\right)_{3} \mathrm{Au}$, using extended Hückel calculations. In the present case, excitation of an electron from the $e^{\prime}$ to $a_{2}{ }^{\prime \prime}$ level amounts to an oxidation of the d orbital manifold, which is then Jahn-Teller unstable, leading to the trigonal planar to T-shape distortion. As a test of our hypothesis, DFT geometry optimization of the doublet $\left[\mathrm{Au}\left(\mathrm{PH}_{3}\right)_{3}\right]^{2+}$ also shows that this structure distorts from trigonal planar to a $\mathrm{T}$-shape.

In summary, this work illustrates that the major excited-state distortion in luminescent $\left[\mathrm{Au}\left(\mathrm{PR}_{3}\right)_{3}\right]^{+}$complexes is a symmetry change as opposed to a bond distance change. This suggests that predictions of the excited-state structure based on the bonding properties of the HOMO and LUMO can be misleading. Such predictions may be valid only if the symmetry of the excited state does not change from that of the ground state. The results of this theoretical study will be used to drive experimental efforts aiming toward (1) chemical and electrochemical oxidation of $\left[\mathrm{Au}\left(\mathrm{PR}_{3}\right)_{3}\right]^{+}$ complexes in an attempt to isolate a ground-state Jahn-Teller distorted T-shaped $\mathrm{Au}(\mathrm{II})$ complex and (2) synthesis of phosphorescent 3-coordinate $\mathrm{Au}(\mathrm{I})$ complexes that are specifically targeted for molecular, dendrimer, and polymer LEDs.

Acknowledgment. We thank the National Science Foundation (CHE-0309811) and the Robert A. Welch Foundation (B-1542) for funding of this research to T.R.C. and M.A.O., respectively.

Supporting Information Available: Full computational details and further quantitative results (PDF). This material is available free of charge via the Internet at http://pubs.acs.org.

\section{References}

(1) Lamansky, S.; Djurovich, P.; Murphy, D.; Abdel-Razzaq, F.; Lee, H.; Adachi, C.; Burrows, P. E.; Forrest, S. R.; Thompson, M. E. J. Am. Chem. Soc. 2001, 123, 4304

(2) Omary, M. A.; Kassab, R. M.; Haneline, M. R.; El Bjeirami, O.; Gabbaï, F. P. Inorg. Chem. 2003, 42, 2176

(3) (a) Mansour, M. A.; Connick, W. B.; Lachicotte, R. J.; Gysling, H. J.; Eisenberg, R. J. Am. Chem. Soc. 1998, 120, 1329. (b) Fernandez, E. J.; Lopez-de-Luzuriaga, J. M.; Monge, M.; Olmos, M. E.; Perez, J.; Laguna, A.; Mohamed, A. A.; Fackler, J. P., Jr. J. Am. Chem. Soc. 2003, 125, 2022.

(4) Mills, A.; Lepre, A.; Theobald, B. R. C.; Slade, E.; Murrer, B. A. Anal. Chem. 1997, 69, 2842

(5) Yam, V. W.-W.; Li, C.-K.; Chan, C.-L. Angew. Chem., Int. Ed. Engl. 1988, 27, 2857.

(6) For a review, see: Yam, V. W.-W.; Lo, K. K. Chem. Soc. Rev. 1999, 28 , 323.

(7) Gimeno, M. C.; Laguna, A. Chem. Rev. 1997, 97, 511

(8) McCleskey, T. M.; Gray, H. B. Inorg. Chem. 1992, 31, 1733

(9) (a) King, C.; Khan, M. N. I.; Staples, R. J.; Fackler, J. P., Jr. Inorg. Chem. 1992, 31, 3236. (b) Forward, J. M.; Assefa, Z.; Fackler, J. P., Jr. J. Am. Chem. Soc. 1995, 117, 9103. (c) Assefa, Z.; Forward, J. M.; Grant, T. A. Staples, R. J.; Hanson, B. E.; Mohamed, A. A.; Fackler, J. P., Jr. Inorg. Chim. Acta 2003, 352, 31 .

(10) (a) Brandys, M.; Puddephatt, R. J. Am. Chem. Soc. 2001, 123, 4839. (b) Yam, V. W.-W.; Lee, W. K. J. Chem. Soc., Dalton Trans. 1993, 2097.

(11) For a review of excited-state distortion in metal complexes, see: Zink, J. I.; Shin, K. S. K. In Advances in Photochemistry; Volman, D. H., Hammond, G. S., Neckers, D. C., Eds.; Wiley: New York, 1991; Vol 16.

(12) The QM calculations utilized the B3PW91 functional using the LANL2DZ basis set augmented with two f-type polarization functions and p-type functions for the valence orbitals of gold and one d-type polarization function for phosphorus. The QM/MM calculations employed the ONIOM methodology.

(13) The blue-shift in the absorption of the $\mathrm{PPhCy}_{2}$ versus the $\mathrm{PPh}_{3}$ complex may be related to the elongation of one of the $\mathrm{Au}-\mathrm{P}$ bonds in the bulky $\mathrm{PPhCy}_{2}$ complex. Data in support of this phenomenon include QM/MM calculations on $\left[\mathrm{Au}\left(\mathrm{PCy}_{3}\right)_{3}\right]^{+}$, which show complete dissociation of one ligand, consistent with experimental data.?

(14) The exact peak position depends on temperature, phase, solvent, and $\mathrm{pH}$. The emission peaks are usually very broad (fwhm $=$ several thousand $\left.\mathrm{cm}^{-1}\right)$.

(15) Komiya, S.; Albright, T. A.; Hoffmann, R.; Kochi, J. K. J. Am. Chem Soc. 1976, 98, 7255

JA036508U 